NEW ZEALAND JOURNAL OF MATHEMATICS

Volume 52 (2021), 145-152

https://doi.org/10.53733/94

\title{
TRISECTIONS AND LINK SURGERIES
}

\author{
Robion Kirby And Abigail Thompson \\ (Received 12 March, 2021)
}

\begin{abstract}
We examine questions about surgery on links which arise naturally from the trisection decomposition of 4-manifolds developed by Gay and Kirby 3. These links lie on Heegaard surfaces in $\#^{j} S^{1} \times S^{2}$ and have surgeries yielding $\#^{k} S^{1} \times S^{2}$. We describe families of links which have such surgeries. One can ask whether all links with such surgeries lie in these families; the answer is almost certainly no. We nevertheless give a small piece of evidence in favor of a positive answer.
\end{abstract}

We are so pleased to have our paper included in this volume honoring Vaughan Jones. Vaughan was a mathematician that few can equal, but perhaps even more important, he was a fount of energy, very generous with his time and ideas, and a great friend to all of us.

\section{Introduction}

The question of which manifolds can arise from Dehn surgery on a knot in the 3-sphere is much-studied, from Gabai's proof of Property R [2], to Gordon and Luecke's solution to the knot complement problem [5], to the as-yet open question posed by the Berge conjecture [7] regarding which knots have surgeries yielding lens spaces. Expanding our attention to consider multi-component links in the 3 -sphere, obtaining similar results even for 2-component links has proven remarkably difficult. For example, attempting the most straightforward generalization of Property $\mathrm{R}$ to a 2-component link in the 3 -sphere (i.e., characterizing 2-component links with surgeries yielding $\#^{2} S^{1} \times S^{2}$ ) has given rise to potential counter-examples to the slice-ribbon conjecture [4. We suggest a framework inspired by trisections of 4manifolds into which many of these questions can be placed.

In the next section we review basic terminology of trisections and Heegaard splittings. In section 3 we introduce some new definitions and use them to prove two lemmas about link surgeries in arbitrary 3-manifolds yielding some number of copies of $\# S^{1} \times S^{2}$. In section 4 we pose some questions in the specific context arising from trisections, and in section 5 we prove our main result.

\section{Background and Definitions}

Let $X$ be a closed, orientable, smooth 4-manifold. In 3 Gay and Kirby show that $X$ has a trisection into three 4-dimensional handlebodies, and prove that any two trisections of $X$ are stably equivalent under a suitable notion of stabilization.

2020 Mathematics Subject Classification 57M27.

Key words and phrases: trisections; link surgeries; Heegaard splittings.

Both authors are supported in part by the National Science Foundation. 
1. Definition. A $\left(g ; k_{1}, k_{2}, k_{3}\right)$-trisection of a closed, oriented 4-manifold $X$ (where $\left.0 \leq k_{i} \leq g, i=1,2,3\right)$ is a decomposition $X=X_{1} \cup X_{2} \cup X_{3}$, where (1) each $X_{i} \cong \natural^{k_{i}} S^{1} \times B^{3},(2)$ each $X_{i} \cap X_{j} \cong \natural^{g} S^{1} \times B^{2}$ (for $i \neq j$ ), and (3) $X_{1} \cap X_{2} \cap X_{3} \cong$ $\#^{g} S^{1} \times S^{1}$.

The topology of each of the three pieces of $X$ is completely determined by a single integer $k_{i}$, and the topology of each of the overlaps between pieces is determined by another integer $g$. If $k=k_{1}=k_{2}=k_{3}$ the trisection is called balanced. We are particularly interested in balanced trisections with $k=0$.

Given a trisection of $X^{4}$, we have a central surface $\Sigma=X_{1} \cap X_{2} \cap X_{3}$ in $X$ bounding three 3-dimensional handlebodies $X_{i} \cap X_{j}$, which fit together in pairs to form Heegaard splittings of three 3-manifolds in $X$, and these 3 -manifolds in turn uniquely bound three 4 -dimensional 1 -handlebodies. We can thus specify a trisection by considering systems of curves on $\Sigma$ :

2. Definition. A cut system for a closed surface $\Sigma$ of genus $g$ is a collection of $g$ disjoint simple closed curves on $\Sigma$ which cut $\Sigma$ open into a $2 g$-punctured sphere.

3. Definition. A genus $g$ Heegaard diagram for a closed orientable 3-manifold $M$ is a triple $(\Sigma, \alpha, \beta)$, where $\Sigma$ is a closed orientable genus $g$ surface in $M$, each of $\alpha$ and $\beta$ is a cut system for $\Sigma$, and $M$ is constructed by attaching 2-handles to $\Sigma \times I$ along the $\alpha$ s and $\beta$ s (and then capping off with 3-balls).

4. Definition. A $\left(g ; k_{1}, k_{2}, k_{3}\right)$-trisection diagram is a 4 -tuple $(\Sigma, \alpha, \beta, \gamma)$ such that each of $(\Sigma, \alpha, \beta),(\Sigma, \beta, \gamma)$ and $(\Sigma, \gamma, \alpha)$ are genus $g$ Heegaard diagrams of $\#^{k}{ }_{i} S^{1} \times S^{2}, i=1,2,3$ respectively. A trisection diagram for a given trisection $X=X_{1} \cup X_{2} \cup X_{3}$ is a trisection diagram $(\Sigma, \alpha, \beta, \gamma)$, where $\Sigma$ is diffeomorphic to $X_{1} \cap X_{2} \cap X_{3}, \alpha$ is a cut system for $X_{1} \cap X_{2}, \beta$ for $X_{2} \cap X_{3}$, and $\gamma$ for $X_{3} \cap X_{1}$.

\section{Link Surgeries in $N^{3}$ yielding $\#^{k} S^{1} \times S^{2}$}

In the case of a balanced trisection with $k=0$, each of the pairs of cut systems define a copy of $S^{3}$, and we can think of building $M^{4}$ in the following way:

Start with a 4 -ball with boundary $S^{3}$. We can think of this bounding $S^{3}$ as being the 3 -sphere determined by the $\alpha$ and $\beta$ curves.

Do integral framed surgery on the $\gamma$ curves to obtain $S^{3}$ again.

Cap off the resulting object with another 4-ball. (For more details on this construction, see [1], Lemma 4.)

The collection of $g \gamma$ curves in $S^{3}$ are a link $L$ lying on a genus $g$ Heegaard surface $\Sigma$ for $S^{3}$. $L$ is a cut system on $\Sigma$.

What follows are some definitions and observations using this set-up as an inspiration:

Let $L$ be a g-component link imbedded as a cut system on the boundary of a genus $g$ handlebody $H$. We say that $L$ is primitive on $H$ if there exists a complete set of compressing disks for $H$ whose boundaries are geometrically dual to $L$ (see figure 1. top). Note that the boundary of $H$ naturally induces a framing on the components of $L$, the surface framing. We say that $L$ is slide-primitive on $H$ if it is possible to do surface-framed handle slides on $L$ lying within $\partial H$ to obtain a link $L^{\prime}$ such that $L^{\prime}$ is primitive on $H$.

Let $L$ be a g-component link imbedded as a cut system on a genus $g$ Heegard surface $\Sigma$ in a 3 -manifold $N^{3}$, with $\Sigma$ bounding handlebodies $H_{1}$ and $H_{2}$. 


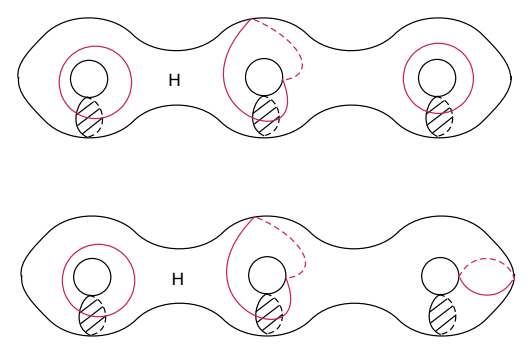

FIGURE 1. primitive system (top) and pseudo-primitve system (bottom)

We say that $L$ is double-slide-primitive (or $d s p$ ) on $\Sigma$ if $L$ is slide-primitive on $H_{1}$ and on $H_{2}$.

5. Lemma. Let $L$ be a g-component link imbedded as a cut system on a genus $g$ Heegard surface $\Sigma$ in a 3-manifold $N^{3}$, with $\Sigma$ bounding handlebodies $\mathrm{H}_{1}$ and $\mathrm{H}_{2}$. Then surface-framed surgery on $L$ yields $S^{3}$ iff $L$ is dsp on $\Sigma$.

Proof:

Assume surgery on $L$ yields a 3 -sphere. The cut system $L$ caps off $\Sigma-L$ into a 2 -sphere after surgery on $L$. Since the result of surgery is $S^{3}$, this 2-sphere must bound a 3 -ball on both sides. Thus each of the manifolds $M_{i}=H_{i} \cup(2$-handles $)$, where the 2-handles are attached along $L$, must be 3 -balls. The result follows from Waldhausen's theorem 13 on Heegaard splittings of the 3-sphere applied to each $M_{i}$.

Conversely if $L$ is dsp on $\Sigma$, then each of the $M_{i}$ are 3-balls and so the surgered manifold is the 3 -sphere.

We can generalize this:

Let $L$ be a g-component link imbedded as a cut system on the boundary of a genus $g$ handlebody $H$. We say that $L$ is pseudo-primitive on $H$ if there exists a complete set of compressing disks for $H$ whose boundaries are geometrically dual to $L$ or isotopic to curves in $L$. We say that $L$ is slide-pseudo-primitive on $H$ if it is possible to do surface-framed handle slides on $L$ to obtain a link $L^{\prime}$ such that $L^{\prime}$ is pseudo-primitive on $H$ (see figure 1 , bottom).

Let $L$ be a g-component link imbedded as a cut system on a genus $g$ Heegard surface $\Sigma$ in a 3-manifold $N^{3}$, with $\Sigma$ bounding handlebodies $H_{1}$ and $H_{2}$.

We say that $L$ is double-slide-pseudo-primitive (or $d s p p$ ) on $\Sigma$ if $L$ is slide-pseudoprimitive on $H_{1}$ and on $H_{2}$.

6. Lemma. Let $L$ be a g-component link imbedded as a cut system on a genus $g$ Heegard surface $\Sigma$ in a 3-manifold $N^{3}$, with $\Sigma$ bounding handlebodies $H_{1}$ and $H_{2}$. Then surface-framed surgery on $L$ yields $\#^{k} S^{1} \times S^{2}$ iff $L$ is dspp on $\Sigma$.

Proof: 
Assume surgery on $L$ yields $\#^{k} S^{1} \times S^{2}$. The cut system $L$ caps off $\Sigma-L$ into a 2 -sphere after surgery on $L$. Since the result of surgery is $\#^{k} S^{1} \times S^{2}$, this 2-sphere must bound $k_{i}, i=1,2$, copies of $\# S^{1} \times S^{2}$ on both sides, where $k_{1}+k_{2}=k$. Thus each of the manifolds $M_{i}=H_{i} \cup(2$-handles $)$, where the 2-handles are attached along $L$, is $\#^{k_{i}} S^{1} \times S^{2}$. The result follows from the generalization of Waldhausen's theorem ( [12], p. 313) on Heegaard splittings of the 3-sphere to Heegaard splittings of $\#^{k_{i}} S^{1} \times S^{2}$.

Conversely if $L$ is dspp on $\Sigma$, then each of the $M_{i}$ are $\#^{k_{i}} S^{1} \times S^{2}$ and so the surgered manifold is $\#^{k} S^{1} \times S^{2}$.

In the context of trisections of 4-manifolds, $N^{3}$ in Lemma 6 is always itself some number of copies, say $j$, of $S^{1} \times S^{2}$. In the next section we consider this case specifically.

\section{Link Surgery Questions arising from Trisections}

Suppose $L$ is a g-component link imbedded as a cut system on a genus $g$ Heegard surface in $\#^{j} S^{1} \times S^{2}$ and surface-framed surgery on $L$ yields $\#^{k} S^{1} \times S^{2}$. Allowing arbitrary handle slides on $L$ will not change the result of the surgery, but may turn $L$ into a link $L^{\prime}$ which no longer lies on the Heegaard surface. One could conjecture that this is essentially the only way to generate such an $L$.

Even more optimistically, in the special case where $N^{3}$ is the 3 -sphere (so $j=k=0$ ) and surgery on $L$ yields the 3 -sphere back, one could hope $L$ is even simpler than slide-equivalent to a "dsp" link on a Heegaard surface, and ask the following:

Question 1: Let $L$ be a $g$ component framed link in the 3 -sphere such that surgery on $L$ yields the 3 -sphere. Is $L$ handle-slide equivalent to a union of Hopf links and unknots?

The answer to this is "no"; Akbulut and Kirby ([1], Figure 29) give a handle decomposition of $K 3$ which utilizes a 22-component link $L$ in the 3 -sphere with integral surgery yielding $S^{3}$. This link cannot be handle-slide equivalent to a union of Hopf links and unknots ( $K 3$ has signature 16 but Hopf links and unknots give signature 0). Lambert-Cole and Meier [9] and Spreer and Tillmann [14] have shown that this decomposition corresponds to a genus 22 balanced trisection of $K 3$ with all sectors a 4-ball. We note that all components of the Akbulut-Kirby link are unknots, so even if we add the hypothesis that each component is unknotted the answer to the question is still no.

We increase our chance of success by asking for less:

Question 2: Let $L$ be a $g$ component framed link in the 3-sphere, such that surgery on $L$ yields the 3 -sphere. Must $L$ be handle-slide equivalent to a dsp link on a genus $g$ Heegaard surface for $S^{3}$ ?

The answer to this is likely also "no", but we don't know any counter-examples. A small piece of evidence in the "yes" direction for this question is our main theorem, which will appear in the final section. 
To conclude this section, we state the most general form of the surgery question as it arises in the trisection context, noting a result which provides support for a negative answer:

Question 3: Let $L$ be a $g \geq k$ component framed link in $\#^{k} S^{1} \times S^{2}$ such that surgery on $L$ yields $\#^{j} S^{1} \times S^{2}$. Must $L$ be handle-slide equivalent to a dspp link on a genus $g$ Heegaard surface for $\#^{k} S^{1} \times S^{2}$ ?

Again presumably the answer is "no"; an example of why this presumption is justified can be found in 4 . There, a 2-component link $L$ in $S^{3}$ (actually a whole family of 2-component links) is shown to yield $\#^{2} S^{1} \times S^{2}$ after 0 -framed surgery. If $L$ were handle-slide equivalent to a dspp link on a genus 2 Heegaard surface for $S^{3}$, it would be handle-slide equivalent to an unlink (4], proposition 3.1). This would imply that conjectured counter-examples to the Andrews-Curtis conjecture fail.

\section{Main Theorem}

7. Theorem. Let $L=L_{1} \cup L_{2}$ be a framed 2-component link in the 3-sphere such that surgery on $L$ yields $S^{3}$. Suppose $L_{1}$ is the unknot.

Then $L$ is handle-slide equivalent to a dsp link on a genus 2 Heegaard surface for $S^{3}$.

A stronger statement follows immediately using a result of Meier and Zupan [10]:

8. Corollary. Let $L=L_{1} \cup L_{2}$ be a framed 2-component link in the 3-sphere such that surgery on $L$ yields $S^{3}$. Suppose $L_{1}$ is the unknot.

Then $L$ is handle-slide equivalent to the unlink or Hopf link.

Proof of Corollary 8 .

Meier and Zupan [10] classify all genus 2 trisection diagrams. The corollary follows from this classification once $L$ has been handle-slid using Theorem 7 to lie on a genus 2 Heegaard surface for $S^{3}$.

Proof of Theorem 7

Do the indicated surgery on $L_{1}$ (see Figure 2). Since $L_{1}$ is the unknot, this yields $M=S^{3}, S^{1} \times S^{2}$ or a lens space $\mathrm{L}(\mathrm{n}, 1)$. $L_{2}$ is then a knot in $M$, and surgery on $L_{2}$ must yield $S^{3}$. If $M$ is $S^{3}$ or $S^{1} \times S^{2}, L_{2}$ is respectively the unknot [5] or a core curve of a genus one Heegaard splitting [2]. The trickiest case is the lens space case. However we are considering only integer surgeries, and the Berge conjecture [7] is known in $\mathrm{L}(\mathrm{n}, 1)[\mathbf{8}$. The Berge conjecture asserts that if surgery on a knot in the 3 -sphere yields a lens space, the knot is doubly-primitive on a genus 2 Heegaard surface in the 3 -sphere. Dually, it asserts that if surgery on a knot in a lens space yields the 3-sphere, the knot has bridge number one with respect to the genus one Heegaard splitting of the lens space. Hence in all cases $L_{2}$ can be isotoped in $M$ to have bridge number zero or one with respect to the genus one Heegaard splitting defined by $L_{1}$ (see Figure 3). During this isotopy, $L_{2}$ may cross the dual knot $L_{1}^{\prime}$. Each such crossing corresponds to a handle-slide of $L_{2}$ over $L_{1}$ in the original link diagram (see the proof of proposition 3.2 in [4]). Once the isotopy is complete (or the corresponding handle slides in the 3 -sphere are complete), the new link $L_{1} \cup L_{2}^{\prime}$ 

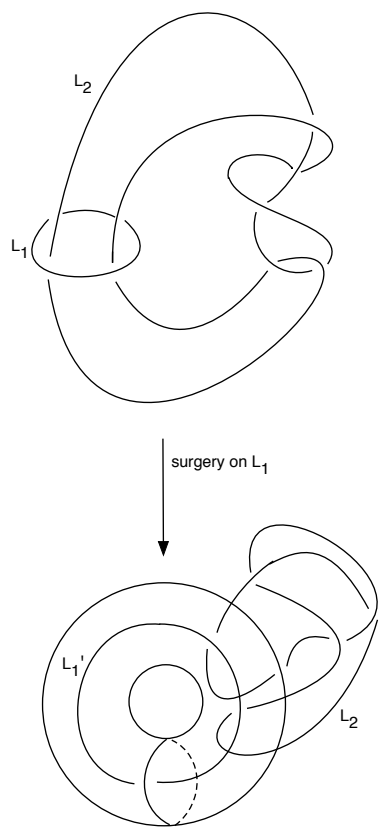

Figure 2.

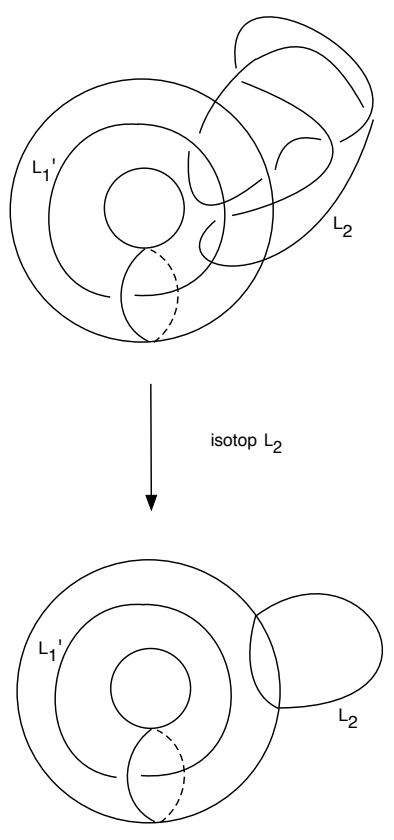

FiguRE 3. 
is tunnel number one, seen by tubing the genus one surface along the arc of $L_{2}$ outside of the handlebody containing $L_{1}^{\prime}$. Hence the link $L^{\prime}=L_{1} \cup L_{2}^{\prime}$ in $S^{3}$ can be imbedded as required (i.e., as a cut system with the desired framing) on a genus 2 Heegaard surface in $S^{3}$. The theorem follows from Lemma 5.

\section{Acknowledgement}

We thank Jeff Meier for many helpful comments resulting in considerable simplification of the main result.

\section{References}

[1] S. Akbulut and R. Kirby, Branched covers of surfaces in 4-manifolds, Math. Ann. 252 (1980), 111-131.

[2] D. Gabai Foliations and the topology of 3-manifolds II, J. Diff. Geom., vol. 26, no. 3 (1987), 461-478.

[3] D.T. Gay and R. Kirby, Trisecting 4-manifolds, Geom. Topol., 20 (2016), 3097-3132.

[4] R. Gompf, M. Scharlemann and A. Thompson, Fibered knots and potential counterexamples to the Property $2 R$ and Slice-Ribbon Conjectures, Geom. Topol., vol. 14, no. 4 (2010), 2305-2347.

[5] C. McA. Gordon and J. Luecke, Knots are determined by their complements, Bull. Amer. Math. Soc. (N.S.), vol. 20, no. 1 (1989), 83-87.

[6] W. Haken, Some results on surfaces in 3-manifolds, Studies in Modern Topology, Math. Assoc. Amer., Prentice-Hall, (1968), 39-98.

[7] R. Kirby, Problems in low-dimensional topology, https://math.berkeley. edu/ kirby/, problem 1.78, (2010).

[8] P. Kronheimer,T. Mrowka, P. Ozsvath, Z. Szabo, Monopoles and lens space surgeries, Annals of Mathematics, 165 (2007), 457-546.

[9] P. Lambert-Cole and J. Meier, Bridge trisections in rational surfaces, Journal of Topology and Analysis, (2020).

[10] J. Meier and A. Zupan, Genus-two trisections are standard, Geom. Topol., 21 (2017), 1583-1630.

[11] J. Meier and A. Zupan, Characterizing Dehn surgeries on links via trisections, PNAS, 43 (2018), 10887-10893.

[12] S. Schleimer, Waldhausen's Theorem, Geometry \& Topology Monographs 12 (2007), 299-317

[13] F. Waldhausen, Heegaard-Zerlegungen der 3-Sphäre, Topology 7 (1968), 195-203.

[14] J. Spreer and S. Tillmann The trisection genus of standard simply connected PL 4-manifolds, 34th International Symposium on Computational Geometry (2018). 
Robion Kirby

Department of Mathematics

University of California

Berkeley

California, USA.

rckirby@berkeley.edu
Abigail Thompson

Department of Mathematics

University of California

Davis

California, USA.

thompson@math.ucdavis.edu 